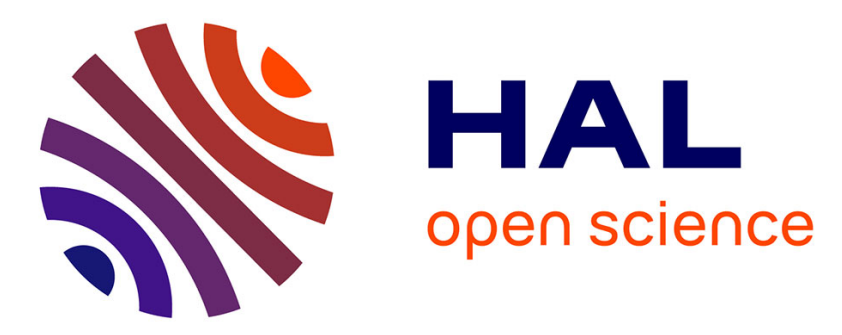

\title{
Surface regularity via the estimation of fractional Brownian motion index
}

Hamed Rabiei, Olivier Coulon, Julien Lefèvre, Frédéric Jp Richard

\section{To cite this version:}

Hamed Rabiei, Olivier Coulon, Julien Lefèvre, Frédéric Jp Richard. Surface regularity via the estimation of fractional Brownian motion index. IEEE Transactions on Image Processing, inPress, 10.1109/TIP.2020.3043892 . hal-02512954

\section{HAL Id: hal-02512954 \\ https://hal.science/hal-02512954}

Submitted on 20 Mar 2020

HAL is a multi-disciplinary open access archive for the deposit and dissemination of scientific research documents, whether they are published or not. The documents may come from teaching and research institutions in France or abroad, or from public or private research centers.
L'archive ouverte pluridisciplinaire HAL, est destinée au dépôt et à la diffusion de documents scientifiques de niveau recherche, publiés ou non, émanant des établissements d'enseignement et de recherche français ou étrangers, des laboratoires publics ou privés. 


\title{
Identification of fractional Brownian motions indexed on surfaces*
}

\author{
Hamed Rabiei $^{\dagger \ddagger} \quad$ Olivier Coulon $^{\ddagger} \quad$ Julien Lefèvre ${ }^{\ddagger} \quad$ Frédéric J.P. Richard ${ }^{\dagger \S}$
}

March 20, 2020

\begin{abstract}
The recent definition of fractional Brownian motions on surfaces has raised the statistical issue of estimating the Hurst index characterizing these models. To deal with this open issue, we propose a method which is a based on a spectral representation of surfaces built upon their Laplace-Beltrami operator. This method includes a first step where the surface supporting the motion is recovered using a mean curvature flow, and a second one where the Hurst index is estimated by linear regression on the motion spectrum. The method is evaluated on synthetic surfaces. The interest of the method is further illustrated on some fetal cortical surfaces extracted from MRI as a means to quantify the brain complexity during the gestational age.
\end{abstract}

keywords fractional Brownian motions on surfaces, Laplace-Beltrami operator, Hurst index estimation, fetal cortical surface.

\section{Introduction}

The introduction of the fractional Brownian motion (fBm) by Mandelbrot and Van Ness [25, 27] has inspired a great deal of research towards the modeling of phenomena of an irregular nature. Initially defined in $\mathbb{R}$, this random process has been extended to $\mathbb{R}^{d}$ to give birth to a wide variety of random fields accounting for complex spatial phenomena (see, for instance, [12, 36, 5, 29, 44] or [15] for an exhaustive survey). Besides, there is still an active research to find appropriate definitions of $\mathrm{fBm}$ on non-Euclidean spaces such as surfaces $[22,23,43,17,18]$. In this paper, we focus on this kind of fBm following the definition approach proposed in $[17,18]$.

On the interval $[0,1]$, the $\mathrm{fBm}$ can be defined by a Fourier expansion

$$
B=C \sum_{l=1}^{+\infty} \xi_{l} \lambda_{l}^{-\left(\frac{1}{4}+\frac{H}{2}\right)} \phi_{l},
$$

where $C$ is a positive constant, $H \in(0,1)$ the so-called Hurst index, $\left(\xi_{l}\right)_{l}$ a series of independent, centered, standard and normally distributed random variables, $\left(\phi_{l}\right)_{l}$ an orthogonal basis of $L^{2}(I ; \mathbb{R})$ composed of eigenfunctions of the Dirichlet-Laplacian operator $\Delta$, and $\lambda_{l}$ the eigenvalue of $\Delta$ associated to the eigenfunction $\phi_{l}$. In $[17,18]$, authors proposed a construction of a $\mathrm{fBm} R$ on an arbitrary compact connected surface $S$ of $\mathbb{R}^{3}$ without boundaries using an analogous expansion

$$
R=C \sum_{l=1}^{\infty} \xi_{l} \lambda_{l}^{-\left(\frac{3}{4}+\frac{H}{2}\right)}\left(\phi_{l}-\phi_{l}(o)\right),
$$

where $o$ is a fixed point on $S$, and $\left(\lambda_{l}, \phi_{l}\right)$ are couples of eigenvalue and eigenfunction of the LaplaceBeltrami (LB) operator defined on $S$ (see [8] and Section 2 for a definition). As for the original fBm,

${ }^{*}$ This work was funded by the Labex Archimède.

${ }^{\dagger}$ Aix-Marseille University, CNRS, Centrale Marseille, I2M, Marseille, France

${ }^{\ddagger}$ Institut de Neurosciences de la Timone, Aix-Marseille University, CNRS, Marseille UMR 7289, France

${ }^{\S}$ Corresponding author: frederic.richard@univ-amu.fr, https://www.i2m.univ-amu.fr/perso/frederic.richard/. 


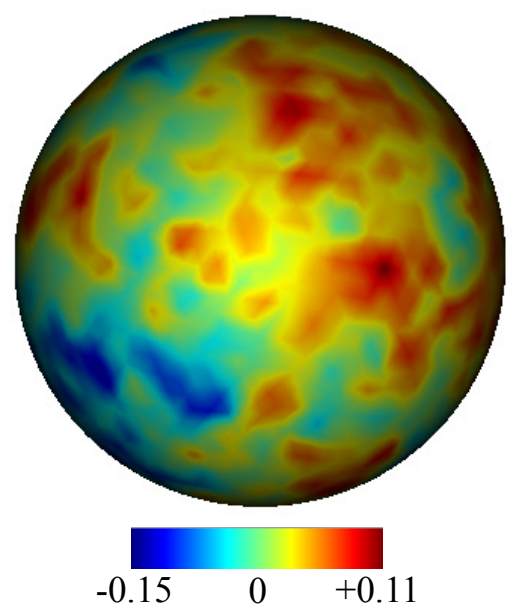

(a) $H=0.1$

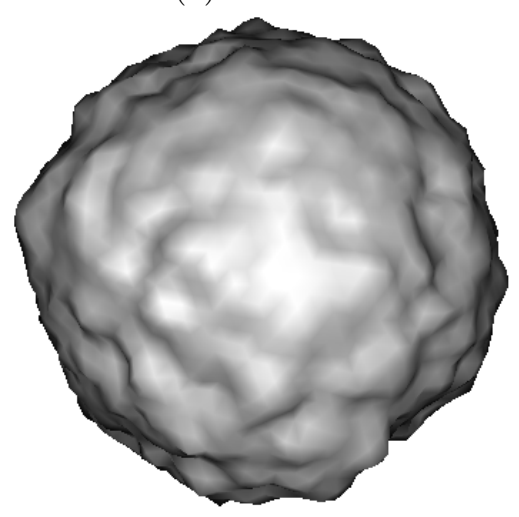

(d) $H=0.1$

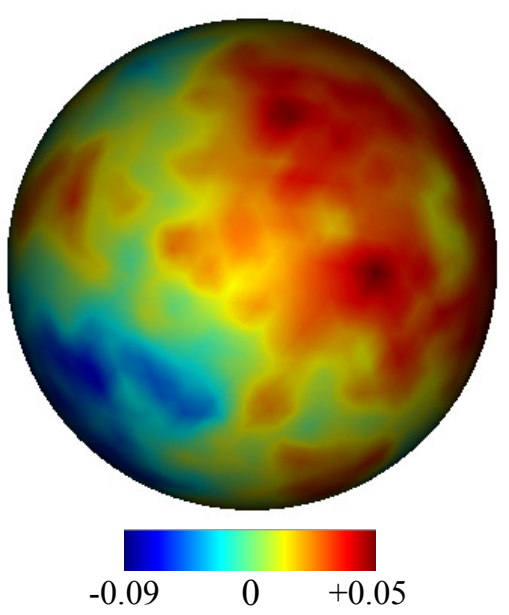

(b) $H=0.5$

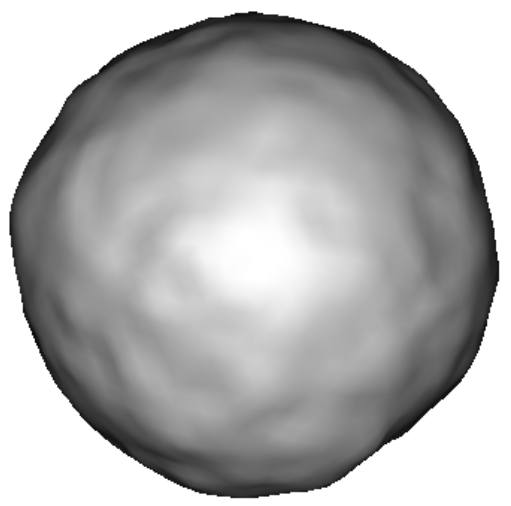

(e) $H=0.5$

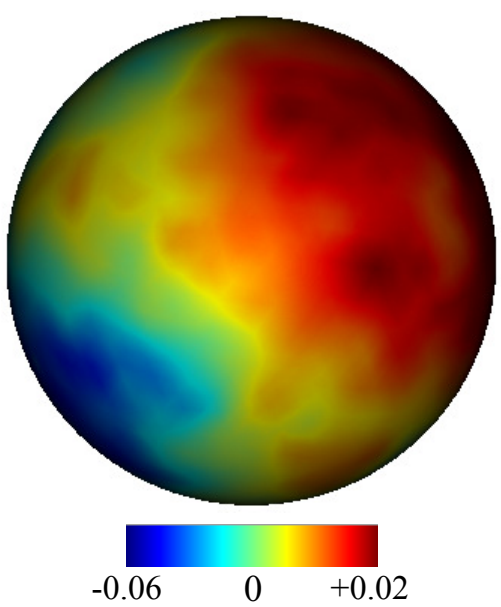

(c) $H=0.9$

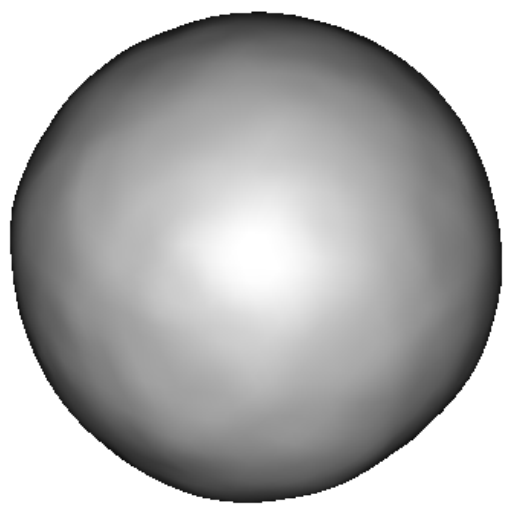

(f) $H=0.9$

Figure 1: Top row: examples of an fBm defined on a sphere. Bottom row: Corresponding fBs.

the obtained $\mathrm{fBm}$ is self-similar with stationary increments, and has realizations whose regularity is almost surely Hölder of order $H$. Given a $\mathrm{fBm} R$ on $S$, a so-called fractional Brownian surface (fBs)

$$
S^{\mathrm{ob}}=S+R N
$$

could be further defined by deforming $S$ in the direction of its outer unit normals $N$ [18]. Some simulations of $\mathrm{fBm}$ defined on the sphere and their associated fBs are shown on Figure 1 for different values of $H$.

There is a huge statistical literature about the estimation of the Hurst index of the original $\mathrm{fBm}$ (see $[7,14,15]$ for review) and its extensions in $\mathbb{R}^{d}$ (see, for instance, $[4,37,44]$ ), ranging from spectral methods to filtering methods such as quadratic variations or wavelet transforms. By contrast, to the best of our knowledge, there is not any method available for the estimation of the Hurst index of a $\mathrm{fBm}$ defined on a surface. Hence, the main contribution of the paper is the construction of a first method that enables to estimate the Hurst index $H$ of a surface $S^{\text {ob }}$ from one of its realizations.

One of the spectral methods commonly used for the original $\mathrm{fBm} B$ relies upon the so-called periodogram [7] defined as

$$
P_{N}(\lambda)=\frac{1}{2 \pi N}\left|\sum_{t=1}^{N} B(t) e^{i t \lambda}\right|^{2}
$$

for frequencies $\lambda=\frac{2 k \pi}{N}$ with $k=0, \cdots, N-1$. This periodogram can be seen an estimator of the spectral density of the increments of the motion $B$. Due to the behavior of this density, it can be shown that, when $\lambda$ is close to 0 ,

$$
\log \left(\mathbb{E}\left(P_{N}(\lambda)\right)\right)=C+(1-2 H) \log (|\lambda|)+\varepsilon(\lambda),
$$


for some constant $C$, and random Gaussian variables $\varepsilon(\lambda)$. As a result, the Hurst index $H$ can be estimated by a linear regression of the log-periodogram on the log-frequency. In this paper, this estimation method is extended to the case when the $\mathrm{fBm}$ is defined on a surface. This extension is not straigthforward: in the Euclidean case, the support of the motion is set in the definition and known. By contrast, in the surface case, the reference surface $S$ indexing the motion is unobserved. Hence, this reference surface has to be estimated prior to any estimation procedure. The method we propose is based on a spectral representation of the surface built upon its LB operator. It includes an initial recovery of the reference surface followed by a regression procedure on the motion spectrum.

\section{Estimation method}

In this section, we present our method for the estimation of the Hurst index of a fBs $S^{\text {ob }}$ (see Equation 2 ). The method has two main steps, a first one where the reference surface $S$ and the Brownian motion $R$ are both estimated from the observed surface $S^{\text {ob }}$, and a second one where the Hurst index is estimated using a Fourier representation of $R$ on $S$. The whole method is summarized in Algorithm (1) and visualised on Figure 2. Each algorithm step is described into details in the next subsections.
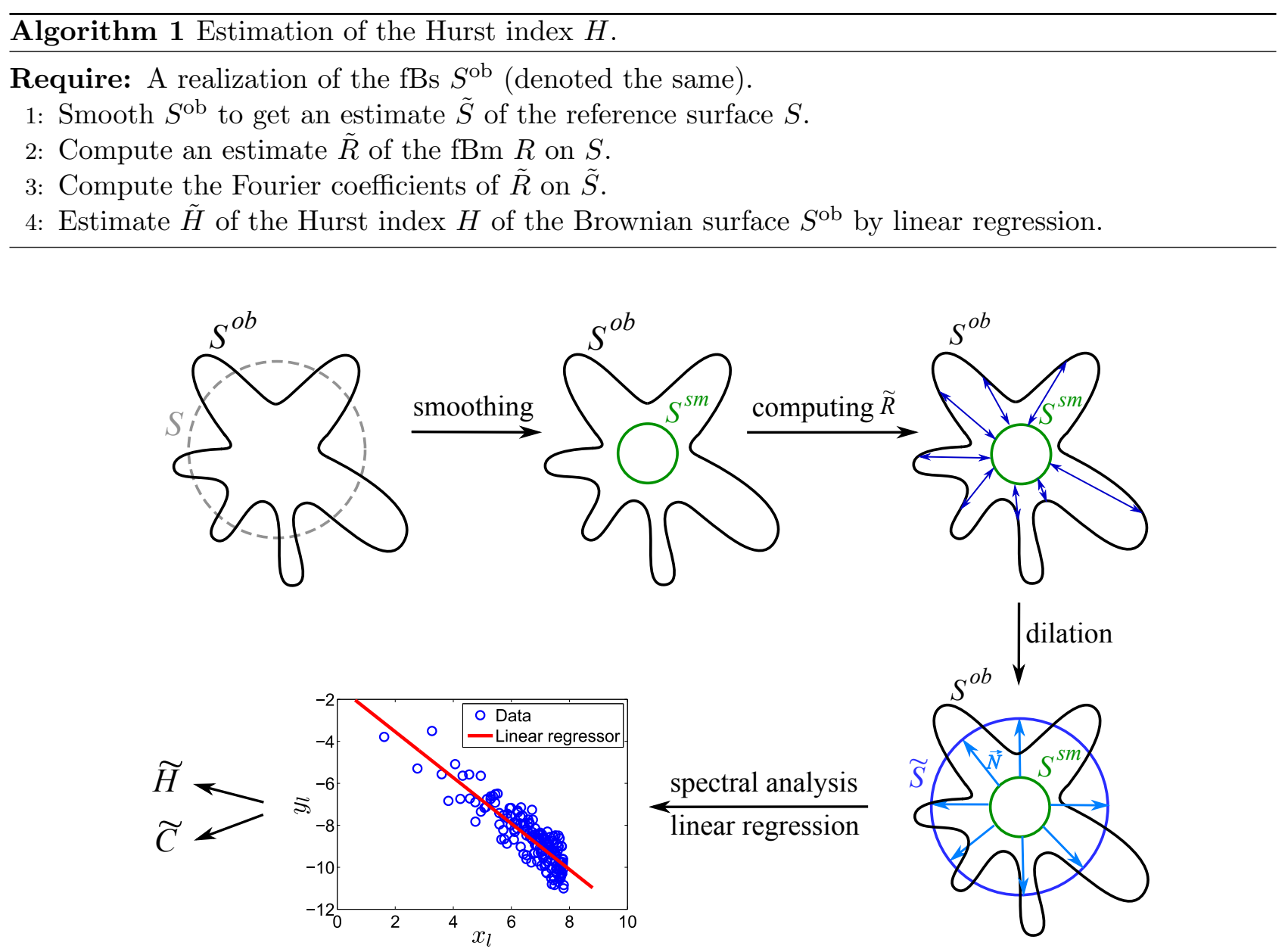

Figure 2: A schematic representation of Algorithm 1. The input to the algorithm is an observed fBs $\left(S^{\text {ob }}\right)$ and output is an estimate of the Hurst index $H$ of the $\mathrm{fBm}$.

\subsection{The LB operator and its discretization}

The estimation method, as well as the surface model, relies upon the LB operator of a surface. In this part, we set up the framework in which this method is designed. 
For a compact Riemannian manifold $S$ in $\mathbb{R}^{3}$, one can define a space of square integrable functions defined on $S$

$$
\mathcal{L}^{2}(S)=\left\{u: S \rightarrow \mathbb{R}, \int_{S} u^{2}<\infty\right\}
$$

and equip it with the inner product $\langle\cdot, \cdot\rangle$ defined for all $u, v \in \mathcal{L}^{2}(S)$ by $\langle u, v\rangle=\int_{S} u v \mathrm{~d} s$.

The LB operator $\Delta$ on $S$ is an extension of the definition of the Dirichlet-Laplacian operator on the non-Euclidian space $S$. It is defined as a combination of the gradient and divergence operators on $S$, namely $\Delta=$ div $\circ$ grad. The spectrum of this operator $\left\{\left(\lambda_{k}, \phi_{k}\right) \in \mathbb{R}^{+} \times \mathcal{L}^{2}(S), k=1,2, \ldots\right\}$ is defined as the set of solutions of the differential eigenvalue problem [8]

$$
\Delta \phi_{k}=-\lambda_{k} \phi_{k}
$$

It is at the basis of the definition of the $\mathrm{fBm}$ on $S$ in Model 1. Using a Green Formula, the spectral problem above can be restated in a weak form

$$
\left\langle\nabla \phi_{k}, \nabla \phi_{i}\right\rangle=-\lambda_{k}\left\langle\phi_{k}, \phi_{i}\right\rangle, \quad \forall \phi_{i} \in \mathcal{L}^{2}(S) .
$$

In practice, we deal with discrete meshes rather than compact surfaces. Hence, for an implementation purpose, we discretize the eigenvalue problem stated above using a finite element method [32]. Let $\mathcal{G}=\{\mathcal{V}, \mathcal{E}\}$ be a mesh composed of a set $\mathcal{V}=\left\{P_{1}, \cdots, P_{N}\right\}$ of $N$ vertices on $S$ and a set $\mathcal{E}$ of edges between these vertices. On $\{1, \cdots, N\}$, we define a space of square integrable functions

$$
l^{2}(\mathcal{V})=\left\{u:\{1, \cdots, N\} \rightarrow \mathbb{R}, \sum_{n=1}^{N} u^{2}(n)<\infty\right\},
$$

where $u(n)$ represents the value of a function $u$ at vertex $P_{n}$. The space $l^{2}(\mathcal{V})$ can be equipped with the inner product defined, for all $u, v \in l^{2}(\mathcal{V})$, by $\langle u, v\rangle=\sum_{n=1}^{N} u(n) v(n)$.

We define a linear function $\psi_{j}$ on the mesh vertices such that, for every vertex $P_{n}, \psi_{j}(n)=\delta_{j n}$, where $\delta_{j n}$ is the Kronecker symbol. In this way, the function $\phi_{k}$ can be interpolated by a linear combination $\sum_{j=1}^{N} \eta_{k j} \psi_{j}$ of $\psi_{j}$ defined with coefficients $\eta_{k j}=\phi_{k}\left(P_{j}\right)$. Substituting this interpolant in the weak form (4), we get

$$
\sum_{j=1}^{N} \eta_{k j}\left\langle\nabla \psi_{j}, \nabla \psi_{i}\right\rangle=-\lambda_{k} \sum_{j=1}^{N} \eta_{k j}\left\langle\psi_{j}, \psi_{i}\right\rangle,
$$

In a matrix form, this is equivalent to the following algebraic generalized eigenvalue equation

$$
A \eta_{k}=\lambda_{k} B \eta_{k}
$$

where $\eta_{k}=\left[\eta_{k 1}, \cdots, \eta_{k N}\right]^{T}$, and $A$ and $B$ are $N \times N$ sparse matrices with the following elements:

$$
A(i, j)= \begin{cases}\frac{\cot \alpha_{i j}+\cot \beta_{i j}}{2} & \text { if }(i, j) \in E, \\ -\sum_{n \in \mathcal{N}(i)} A(i, n) & \text { if } i=j, \\ 0 & \text { o.w. }\end{cases}
$$

and

$$
B(i, j)=\left\{\begin{array}{lc}
\frac{\left|t_{1}\right|+\left|t_{2}\right|}{22} & \text { if }(i, j) \in E, \\
\frac{\sum_{n \in \mathcal{N}(i)}\left|t_{n}\right|}{6} & \text { if } i=j, \\
0 & \text { o.w. }
\end{array}\right.
$$

where $\alpha_{i j}$ and $\beta_{i j}$ are the angles opposite to the edge $P_{i} P_{j}$ in two triangles $t_{1}$ and $t_{2}$ sharing this edge, $\left|t_{n}\right|$ indicates the area of the triangle $t_{n}$ and $\mathcal{N}(i)$ denotes the index set of all vertices of the 1-ring neighbourhood of $P_{i}$.

Being symmetric and positive definite, the matrix $B$ admits a Choleski decomposition $B=B^{\frac{1}{2}} B^{\frac{1}{2}}$. Thus, setting new coordinates $\varphi_{k}=B^{\frac{1}{2}} \eta_{k}$, Problem 6 becomes

$$
B^{-\frac{1}{2}} A B^{-\frac{1}{2}} \varphi_{k}=\lambda_{k} \varphi_{k}
$$


Hence, it can be solved by finding pairs of eigenvalues and eigenvectors $\left(\lambda_{k}, \varphi_{k}\right)$ of the matrix $B^{-\frac{1}{2}} A B^{-\frac{1}{2}}$. Further imposing that eigenvectors $\eta_{k}$ are orthonormal with respect to the inner product $\langle B \cdot, \cdot\rangle$, solutions $\varphi_{k}=B^{\frac{1}{2}} \eta_{k}$ are orthonormal with respect to the usual Euclidean inner product.

Besides, due to the Weyl's theorem [45], the contribution of $M$ th term in Equation (1) is of order $O\left(M^{-(d / 4+H / 2)}\right)$, which becomes negligible for large $M$ 's and fine triangulation. So, in practice, the fractional Brownian random field of Equation (1) can be approximated on a discrete mesh by a field denoted $R_{M}$ having

$$
\rho=C \sum_{l=2}^{M}\left(\lambda_{l}\right)^{-\left(\frac{d}{4}+\frac{H}{2}\right)}\left(\varphi_{l}-\varphi_{l}\left(P_{o}\right)\right) \xi_{l},
$$

as interpolation coefficients and a vertex $P_{o}$ as an origin.

\subsection{Recovery of the reference surface}

To get an estimate of the reference surface $S$, we first smooth the observed surface $S^{\text {ob }}$ by applying a mean curvature flow [21]. Such a procedure relies upon the definition of a heat equation involving a $2 \mathrm{D}$ manifold $\Omega_{t}$ which evolves with time $t$ :

$$
\begin{array}{rrr}
\frac{\partial f}{\partial t}=\Delta_{t} f, & \text { on } \Omega_{t} \times(0, T], \\
\left.f\right|_{t=0}=f^{0}, & \text { on } \Omega_{t},
\end{array}
$$

where $f$ stands for some coordinate components of $\Omega_{t}$, and $\Delta_{t}$ for the LB operator on the manifold $\Omega_{t}$. Starting from the initial state $\Omega_{0}$, points of $\Omega_{t}$ move in the direction of the inner normal to a distance which is proportional to the mean curvature. These displacements tend to smooth $\Omega_{t}$ as the time $t$ evolves. A schematic representation of this procedure is given in Figure 3. Let us quote that it establishes a one-to-one correspondence between points of the initial surface $\Omega_{0}$ and those of the successive smooth surfaces $\Omega_{t}$.

So as to be solved numerically, Equation (11) is discretized in time using a first order backward finite difference scheme and in space using the discretization of the LB operator introduced in the previous section. This leads to a discrete heat equation of the form

$$
(B+d t A) f^{m+1}=B f^{m},
$$

where $f^{m}$ denotes $f$ at a time $t_{m}=m \times d t$, and $A$ and $B$ are matrices given by (7) and (8), respectively. This approach is known to be numerically stable even for large time steps and convergent, at least for convex surfaces [21].

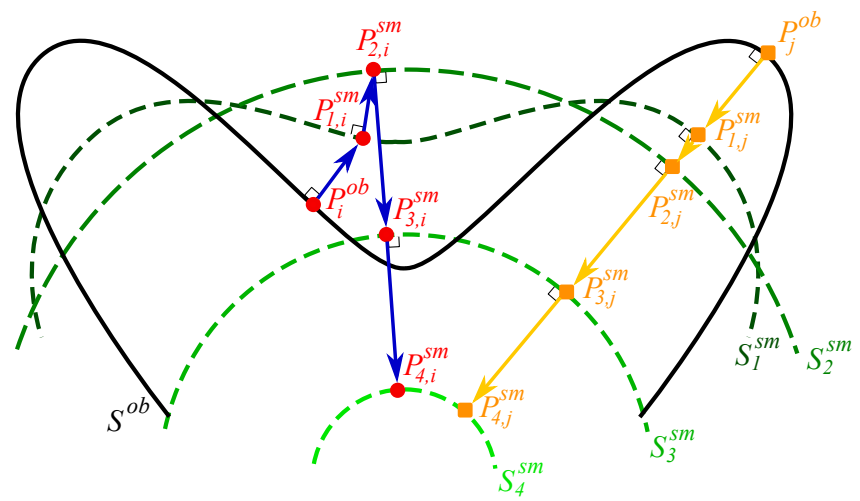

Figure 3: The mean curvature flow smoothing procedure applied to a surface. Geodesic paths of two points $P_{i}^{\mathrm{ob}}$ and $P_{j}^{\mathrm{ob}}$ to their corresponding points on the final smoothed surface are shown in blue and yellow colors, respectively.

The MCF is applied to the observed surface $S^{\mathrm{ob}}$ by setting $\Omega_{0}=S^{\mathrm{ob}}$. It is stopped at a time $t^{*}$ when $\Omega_{t^{*}}$ is entirely inside $S^{\mathrm{ob}}$. Pratically, this stopping condition is considered as fulfilled when all angles between the vector $P_{n}^{\mathrm{sm}} P_{n}^{\mathrm{ob}}$ and the normal vector to $\Omega_{t^{*}}$ at $P_{n}^{\mathrm{sm}}$ are all acute. 
As the MCF tends to move the barycenter of the surface $\Omega_{s}$, the barycenter of $\Omega_{t}$ is aligned to the one of $S^{\mathrm{ob}}$ at each time $t$. Moreover, so as to compensate for a surface shrunking, the final surface $\Omega_{t^{*}}$ is dilated with an iterative procedure that avoids the creation of surface singularities such as point crossing. At each iteration of this procedure, problematic mesh triangles with too acute angles are identified and kept fixed while other triangles are slightly dilated. The holes left by problematic triangles are then filled by a linear interpolation and faired by a mean curvature flow.

In this way, we obtain an estimate $\tilde{S}$ of the reference surface $S$. We could then estimate pairs $\left\{\left(\tilde{\lambda}_{k}, \tilde{\phi}_{k}\right)\right\}_{k=2}^{M}$ of eigenvalues and eigenvectors of the LB operator on $S$ by solving Equation (6) with $A$ and $B$ derived from $\tilde{S}$.

The procedure is pursued by an approximation of the $\mathrm{fBm} R_{M}$. According to Model (2), the value $R_{M}(P)$ of the field $R_{M}$ at some vertex $P$ of $S$ depends on the distance between the vertex $P$ and its corresponding vertex $P^{\mathrm{ob}}$ on $S^{\mathrm{ob}}$. At a vertex $\tilde{P}_{n}$ of the estimated reference surface $\tilde{S}$, it is approximated by

$$
\tilde{R}_{M}(n)=w(n)-\bar{w},
$$

where $w(n)=d\left(P_{n}^{\mathrm{ob}}, \tilde{P}_{n}\right)$ is the Euclidean distance between $P_{n}^{\text {ob }}$ and $\tilde{P}_{n}$ vertices, and $\bar{w}=\frac{1}{N} \sum_{n=1}^{N} w(n)$ is a mean distance. The resulting estimate of $R_{M}$ is zero-mean, which is consistent with its theoretical definition.

\subsection{Spectral estimation of the Hurst index}

According to Model (10), Fourier coefficients of a discrete fBm on a surface mesh are given by

$$
\hat{\rho}_{k}=\left\langle\rho, \varphi_{k}\right\rangle=C \sum_{l=2}^{M} \lambda_{l}^{-\left(\frac{d}{4}+\frac{H}{2}\right)}\left(\left\langle\varphi_{l}, \varphi_{k}\right\rangle-\varphi_{l}\left(P_{o}\right)\left\langle\mathbf{1}, \varphi_{k}\right\rangle\right) \xi_{l},
$$

where $\left(\lambda_{l}, \varphi_{l}\right)_{l}$ are couples of eigenvalue and eigenvector of the LB operator of the surface mesh. Due to the orthogonality of eigenfunctions, Fourier coefficients reduce to

$$
\hat{\rho}_{k}=C \lambda_{k}^{-\left(\frac{d}{4}+\frac{H}{2}\right)} \xi_{k},
$$

for $k=2, \cdots, M$. Consequently,

$$
\log \mathbb{E}\left[\hat{\rho}_{k}^{2}\right]=-\left(\frac{d}{2}+H\right) \log \left(\lambda_{k}\right)+\log \left(C^{2}\right), \quad k=2, \ldots, M .
$$

This equation shows a linear relationship between $\log \mathbb{E}\left[\hat{\rho}_{k}^{2}\right]$ and $\log \left(\lambda_{k}\right)$.

This relationship suggests to estimate $H$ and $C$ from the slope and intercept of a least square linear regression line computed on points $\left\{\lambda_{k}, \mathbb{E}\left[\hat{\rho}_{k}^{2}\right]\right\}$ in log-log scale. For that, an estimate of the expectation term $\mathbb{E}\left[\hat{\rho}_{k}^{2}\right]$ would be required. If many realizations of $R$ were available, such an estimate could be obtained using some laws of large numbers. However, we only have one realization of the $\mathrm{fBm}$.

To deal with this constraint, we shall start with the log-spectrum of $R_{M}$ given by

$$
\log \left(\hat{\rho}_{k}^{2}\right)=-\left(\frac{d}{2}+H\right) \log \left(\lambda_{k}\right)+\log \left(C^{2}\right)+\log \left(\xi_{k}^{2}\right), \quad k=2, \ldots, M,
$$

which follows from Equation (15). To apply a least square linear regression analysis, we propose a binning strategy. Formally, let $\left\{E_{l}, l=1,2, \ldots, L\right\}$ be a partition of spectral indices $\{2,3, \ldots, M\}$, and $N_{l}$ be the size of the set $E_{l}$. Taking the averages in both sides of (17) on each bin $E_{l}$, leads to a linear model of the form

$$
y_{l}=\alpha x_{l}+\gamma+\varepsilon_{l}, \quad l=1, \cdots, L,
$$

where

$$
x_{l}=\frac{1}{N_{l}} \sum_{k \in E_{l}} \log \left(\lambda_{k}\right), \quad y_{l}=\frac{1}{N_{l}} \sum_{k \in E_{l}} \log \left(\hat{\rho}_{k}^{2}\right), \quad \varepsilon_{l}=\frac{1}{N_{l}} \sum_{k \in E_{l}} \log \left(\xi_{k}^{2}\right),
$$




$$
\alpha=-\left(\frac{d}{2}+H\right) \text { and } \gamma=\log \left(C^{2}\right) .
$$

Due to the Central Limit Theorem, the distribution of $\varepsilon_{l}$ can be approximated for large $N_{l}$ by a normal distribution of mean $\mu_{0}=\mathbb{E}\left[\log \left(\xi_{1}^{2}\right)\right]$ and variance $\operatorname{Var}\left[\log \left(\xi_{1}^{2}\right)\right] / N_{l}$. Furthermore, since $\left\{\xi_{1}\right\}$ has a standard normal distribution, $\left\{\xi_{1}^{2}\right\}$ has $\chi^{2}$ distribution, and

$$
\mu_{0}=\log (2)+\psi\left(\frac{1}{2}\right)
$$

where $\psi$ is the so-called $\psi$-function, which is the first derivative of the Gamma function [28]. Hence, setting

$$
\beta=\gamma+\mu_{0}
$$

Equation (18) is asymptotically equivalent to

$$
y_{l}=\alpha x_{l}+\beta+\tilde{\varepsilon}_{l}, \quad l=1,2, \ldots, L .
$$

where $\tilde{\varepsilon}_{l}$ are i.i.d of law $\mathcal{N}\left(0, \frac{\sigma_{0}^{2}}{N_{l}}\right)$. Regression parameters $\alpha$ and $\beta$ of this linear model can be estimated by minimizing a weighted least square criterion:

$$
J(\alpha, \beta)=\sum_{l=1}^{L} \frac{N_{l}}{\sigma_{0}^{2}}\left(y_{l}-\alpha x_{l}-\beta\right)^{2} .
$$

This leads to the following estimates

$$
\begin{aligned}
\alpha & =\frac{\sum_{l=1}^{L} \frac{N_{l}}{N-1} x_{l} y_{l}-\left(\sum_{l=1}^{L} \frac{N_{l}}{N-1} x_{l}\right)\left(\sum_{l=1}^{L} \frac{N_{l}}{N-1} y_{l}\right)}{\sum_{l=1}^{L} \frac{N_{l}}{N-1} x_{l}^{2}-\left(\sum_{l=1}^{L} \frac{N_{l}}{N-1} x_{l}\right)^{2}} \\
\beta & =\sum_{l=1}^{L} \frac{N_{l}}{N-1} y_{l}-\alpha \sum_{l=1}^{L} \frac{N_{l}}{N-1} x_{l} .
\end{aligned}
$$

Now, according to (20), $H$ and $C$ can be obtained as

$$
H=\alpha+d / 2, \quad C=\sqrt{\exp \left(\beta-\mu_{0}\right)} .
$$

In our estimation procedure, the regression (23) is defined and applied with the estimates $\tilde{\lambda}_{k}$ and $\tilde{\rho}$ of the LB operator eigenvalues and fractional Brownian field coefficients, respectively.

\section{$3 \quad$ Numerical study}

We evaluated the proposed algorithm of Section 2 on synthetic data. Using the method developed in [18], we simulated a set of 1000 fractional Brownian spheres with Hurst indices $H$ uniformly sampled from a uniform distribution in $(0.05,0.95)$. A triangulated unit sphere with $N=2562$ vertices served as a reference surface $S$. The first $M=1600 \mathrm{LB}$ eigenpairs of $S$ were computed using the linear FEM of (2.1). Then, fBms $R$ on $S$ were generated via (10) with $C=1$. Fractional Brownian spheres were synthesized by displacing vertices of $S$ in direction of their corresponding outer normal vectors by an amount of $R$. Some examples of such surfaces were shown on (1).

For each surface, $H$ was estimated according to (1); the mean curvature flow was applied with $d t=0.1$. The mean bias (mBias) and the root mean square error (rMSE) of the estimates $\tilde{H}_{k}$ of $H_{k}$ at the $k$ th experiment were computed as

$$
\operatorname{mBias}=\frac{1}{N} \sum_{k=1}^{1000}\left(\tilde{H}_{k}-H_{k}\right) \quad \operatorname{rMSE}=\sqrt{\frac{1}{N} \sum_{k=1}^{1000}\left(\tilde{H}_{k}-H_{k}\right)^{2}} .
$$

Results are presented in (1) for different numbers $n$ of bin elements. rMSE of $H$ did not significantly depend on $n$. Both, the mBias and the rMSE were quite low. The rMSE was about 0.06 corresponding to a relative error of $6 \%$. 
Table 1: Estimation of the Hurst index $H$ on 1000 fractional Brownian spheres. The number $n$ refers to the number of bin element used in the regression procedure 23.

\begin{tabular}{lccccc}
\hline \multirow{2}{*}{ Statistics } & \multicolumn{5}{c}{ Binned regression } \\
\cline { 2 - 6 } & $n=50$ & $n=40$ & $n=30$ & $n=20$ & $n=10$ \\
\hline mBias of $\tilde{H}$ & -0.011 & -0.0089 & -0.0069 & -0.0041 & -0.00039 \\
rMSE of $\tilde{H}$ & 0.065 & 0.065 & 0.065 & 0.064 & 0.064 \\
\hline
\end{tabular}
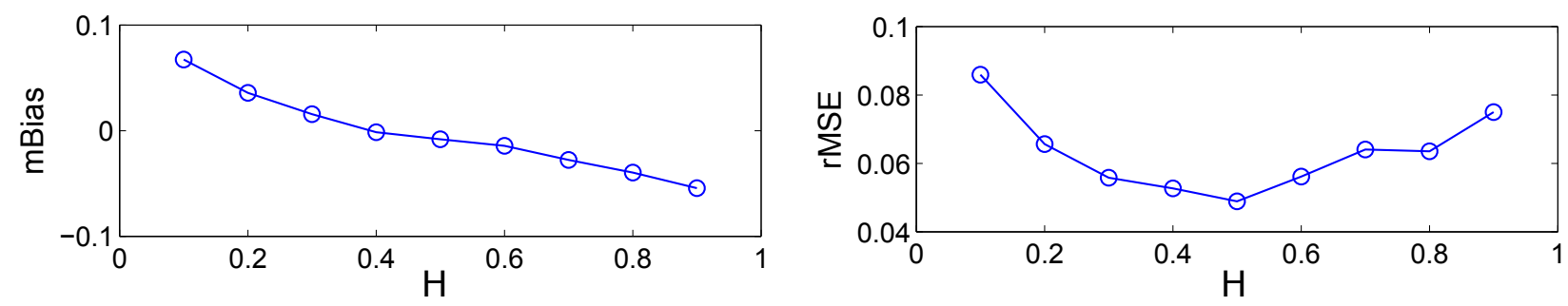

Figure 4: mBias and rMSE of the estimates of the Hurst index $H$ as a function of $H$ values.

The simulated surfaces were further classified into 9 groups depending on their $H$ value: the $i t h$ group contained surfaces with $H \in[0.05+0.1(i-1), 0.05+0.1 i)$ for $i=1,2, \ldots, 9$. For each group, we computed the mBias and rMSE for $n=10$. Results are shown in Figure 4 . We observe that the method tended to overestimate $H$ in the lowest range $(<=0.35)$ and underestimate it in the highest one $(>0.75)$. The maximal rMSE was below 0.1 (10\% error). The most accurate results were achieved for surfaces with medium values of $H$ in $[0.45,0.55)$.

\section{Application to fetal data}

The proposed method was applied to a dataset of fetal cortical surfaces. This dataset was acquired at the Department of Neuroradiology at La Timone Hospital (Marseille, France) between January 1st and December 31st, 2011, in a clinical context and has been used to study the evolution of cortical complexity through gestational development [19]. It consisted of in vivo magnetic resonance images of fetal brains acquired in utero using a 1.5 teslas MR machine, from which cortical surfaces were extracted. Details of the acquisition and preprocessing steps, including image segmentation and 3D reconstruction of surfaces, are explained in [26].

In this dataset, we selected 12 subjects free of diseases according to radiological criteria. The gestational age of these subjects was between 21 and 34 weeks at the time of MRI acquisition (mean age: $29.6 \pm 3.5$ weeks). Thus, we had 24 triangulated hemispheric surfaces.

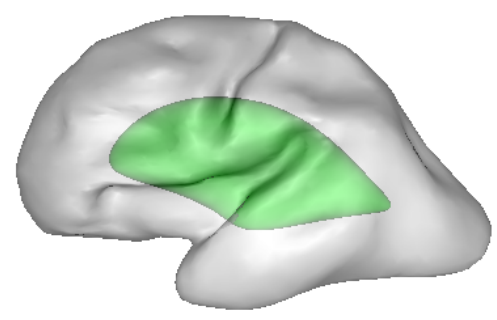

(a)

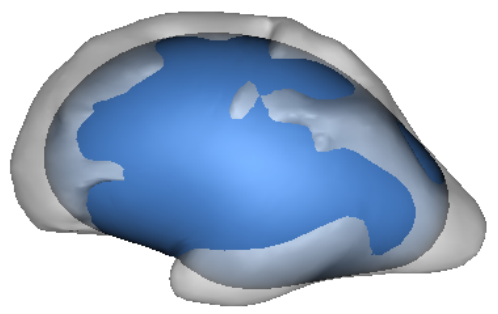

(b)

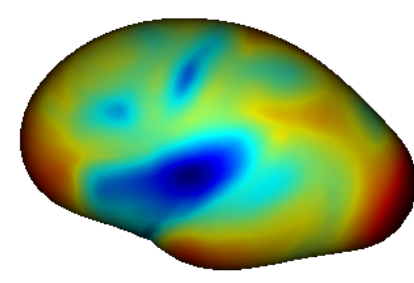

(c)

Figure 5: The first steps of Algorithm 1 applied to a fetal brain hemisphere: (a) the observed surface (in gray) and smooth surface (in green), (b) the estimated reference surface (in blue), (c) the estimated fractional Brownian field on the reference surface. 


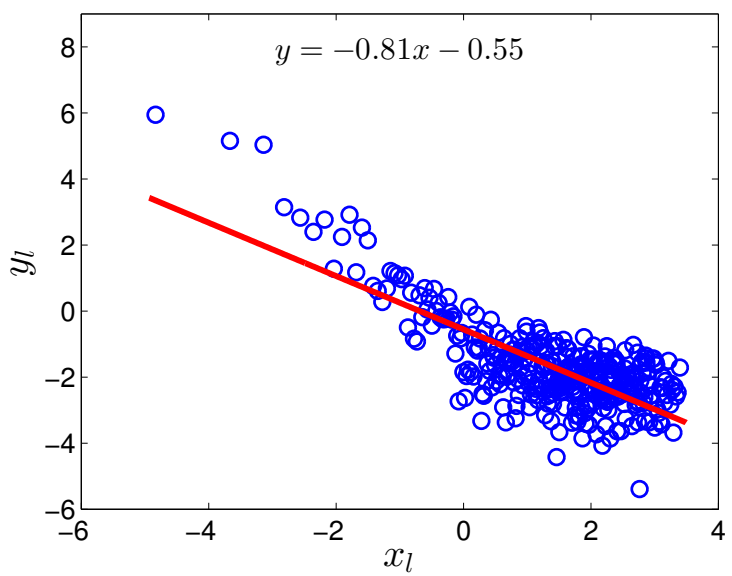

(a)

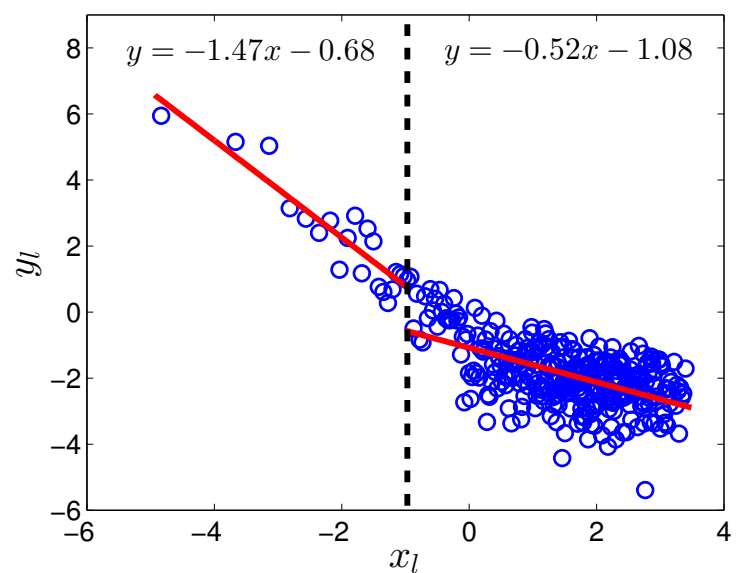

(b)

Figure 6: Linear regression on the spectrum of the cerebral hemisphere of Figure 5 (a) complete regression, (b) partial regressions with the breakpoint $l^{*}=20$.

Considering each brain hemisphere as a realization of a fBs, we applied Algorithm 1 to estimate its Hurst index. The first steps of this algorithm are illustrated on Figure 5a. As explained in Section 2, the estimation of the Hurst index consists of applying a linear regression procedure to the spectrum of the fractional Brownian field on the surface. However, as illustrated on Figure 6a, we observed that relationships between the logarithms of the spectrum energy $y_{l}$ and its corresponding scales (see Equation (19)) was not linear on cortical surfaces. Moreover, when performed on this spectrum, regression analysis could lead to negative Hurst index which were out of the expected range $(0,1)$. This issue could be due to mesh artefacts or numerical inaccuracies of the approximation of large LB eigenpairs. In fact, by using the linear FEM, the absolute error of approximation of LB eigenvalues is bounded by the square of eigenvalues. Moreover, the error of approximation of LB eigenvectors is bounded by corresponding eigenvalue [40]. Hence, as eigenvalues increase, their approximation error may increase, leading to inaccurate spectrum features $x_{l}$ and $y_{l}$.

To fix this issue, a segmented linear regression was performed. In this method, $x_{l}$ 's was broken into two segments. The breakpoint (the border of low and high $x_{l}$ 's) was manually set from the data of the smallest surface in database (having 930 vertices) to $l^{*}=20$ at a point where the linear trend of the first eigenvalues started to be invalided; see illustration on Figure 6b. Let us quote that the approximated reference surfaces of all subjects in the database had similar shapes but different sizes. So, their LB eigenvalues were similar up to a scaling factor [33]: larger surfaces had smaller eigenvalues. So, the $x_{l^{*}}$ value itself could not be used as the breakpoint since it was scaled across subjects and could result in regression analysis in different spectral bands.

On Figure $7 \mathrm{a}$, we show estimates $\tilde{H}$ of the Hurst index of left and right hemispheres as a function of the gestional age. We observe that older subjects tend to have smaller values of $\tilde{H}$. As age increases during the fetal age, folds appear on the brain surface, increasing the complexity of the cortical surface ([26]). So, the decrease of $\tilde{H}$ with age may reflect this increase of complexity.

In the literature, it was observed that larger fetal brains are more folded (e.g. [26]). This was also verified by simulation on some mechanical models $([41,42])$. On Figure $7 \mathrm{~b}$, we plot the value of $\tilde{H}$ as a function of the hemispheric volume. We observe that $\tilde{H}$ decreased as the volume increased. This suggests again that the Hurst index could be a good indicator of surface complexity.

\section{Discussion}

The recent definition of fractional Brownian motions on surfaces $[17,18]$ has raised the issue of estimating the Hurst index which characterizes these new models. In this paper, we proposed an original estimation method which is based on a spectral representation of surfaces built upon their LaplaceBeltrami operator. This method involves two main steps: a first one where a mean curvature flow 


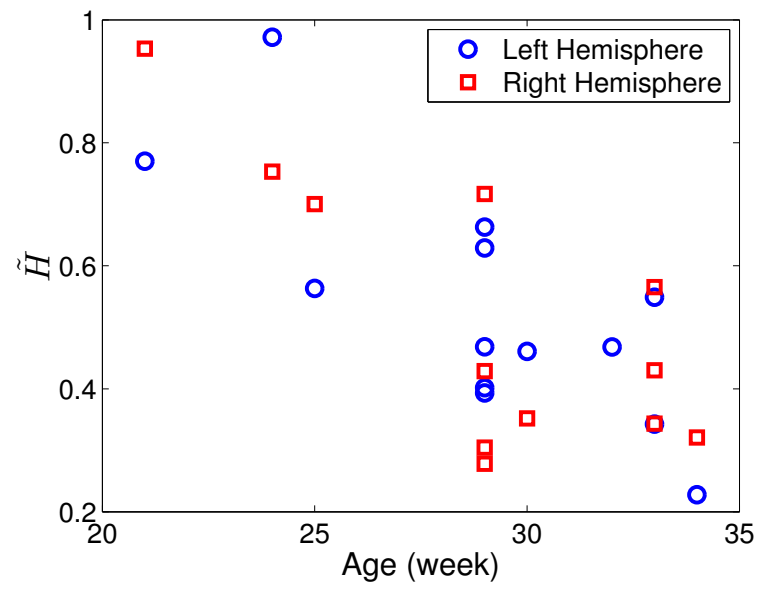

(a)

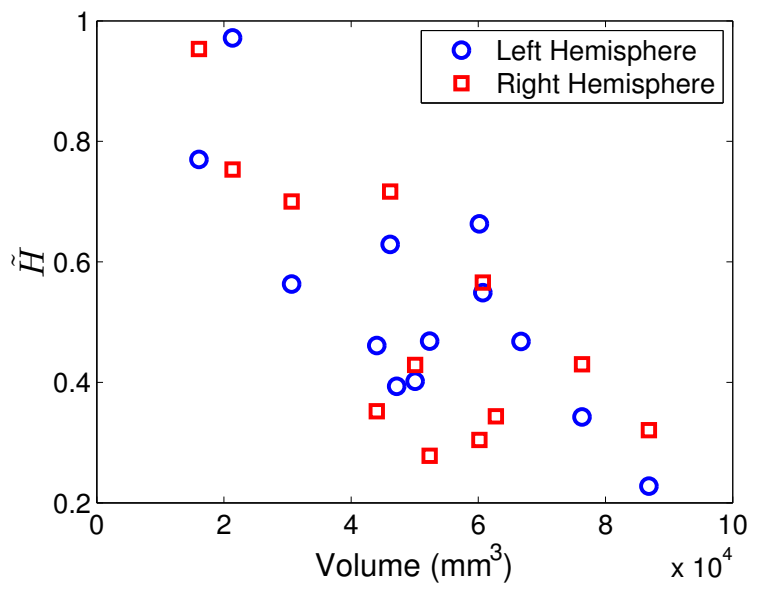

(b)

Figure 7: (a) $\tilde{H}$ versus gestational age, and (b) $\tilde{H}$ versus hemispheric volume of fetal subjects.

is applied to recover the surface indexing the $\mathrm{fBm}$, and a second one where a regression procedure is applied to the $\mathrm{fBm}$ spectrum to identify the Hurst index. The method was tested on simulated surfaces leading to a mean error of about $6 \%$, which is a promising score for a first attempt to solve the open issue.

The method can be seen as an extension of a spectral method currently used for classical fBm. By extending other classical methods, it could also be possible to construct alternate estimation methods for surface-indexed fBm. For instance, the classical filtering methods based on quadratic variations [24] or wavelets [16] could be extended using some recent wavelet representations on graphs [1, 20]. To avoid the effect of numerical inaccuracies of the approximation of large LB eigenpairs, our method was applied considering only the lowest eigenpairs. As a result, the method mainly captured some information about the surface low-frequencies. Using alternate filtering methods, we could focus on surface high-frequencies and complete these information.

The Hurst index is a feature which characterizes the global regularity of a fractional Brownian object (random field or surface) assuming that it is homogeneous. Some heterogeneous random field models (indexed on $\mathbb{R}^{d}$ ) known as multifractional Brownian fields $[5,29,9,2,3,44]$ were defined to account for local variations of the regularity of a field. Some methods were also constructed to estimate the local regularity of these fields. However, it remains an open question to define mathematically some heterogeneous fractional Brownian surfaces with a varying local regularity. Still, our estimation method could be extended to deal with the estimation of the local regularity of heterogeneous surfaces using a local spectrum analysis based on a windowed Fourier transform on surfaces [39, 30, 31].

Besides, the Hurst index is an isotropic features which characterizes the object independently of any direction. The fBs, itself, is an isotropic model that has the same properties whatever the orientation. For random fields, there has been a lot of research devoted to the definition of anisotropic models, as well as the characterization and estimation of their directional properties $[12,6,10,13$, $37,35,36,34,38,11,44]$. Defining and analyzing anisotropic fBs is another path of research that is opened by this work.

\section{References}

[1] L. Avena, F. Castell, A. Gaudillière, And C. MÉlot, Intertwining wavelets or multiresolution analysis on graphs through random forests, Appl. Comput. Harmon. Anal., (2018).

[2] J. Ayache And J. LÉvy-VÉHel, Generalized multifractional Brownian motion: definition and preliminary results, in Fractals: Theory and Applications in Engineering, L. Lévy-Véhel and Tricot, eds., Springer, 1999, pp. 17-32. 
[3] A. Benassi, P. Bertrand, S. Cohen, and J. Istas, Identification of the Hurst index of a step Fractional Brownian motion, Stat. Inference Stoch. Process., 3 (2000), pp. 101-111.

[4] A. Benassi, S. Cohen, And J. Istas, Identifying the multifractional function of a Gaussian process, Statist. Probab. Lett., 39 (1998), pp. 337-345.

[5] A. Benassi, S. Jaffard, And D. Roux, Elliptic Gaussian random processes, Rev. Mathem. Iberoamericana, 13 (1997), pp. 19-89.

[6] D. Benson, M. M. Meerschaert, B. Bäumer, And H. P. Scheffler, Aquifer operatorscaling and the effect on solute mixing and dispersion, Water Resour. Res., 42 (2006), pp. 1-18.

[7] J. Beran, Statistics for long-memory processes, Routledge, 2017.

[8] M. Berger, A Panoramic View of Riemannian Geometry, Springer Berlin Heidelberg, Berlin, Heidelberg, 2003.

[9] H. Biermé, C. Lacaux, And H. Scheffler, Multi-operator scaling random fields, Stoch. Proc. Appl, 121 (2011), pp. 2642-2677.

[10] H. Biermé, M. M. Meerschaert, And H. P. Scheffler, Operator scaling stable random fields, Stoch. Proc. Appl., 117 (2007), pp. 312-332.

[11] H. Biermé And F. Richard, Estimation of anisotropic Gaussian fields through Radon transform, ESAIM: Probab. Stat., 12 (2008), pp. 30-50.

[12] A. Bonami And A. Estrade, Anisotropic analysis of some Gaussian models, J. Fourier Anal. Appl., 9 (2003), pp. 215-236.

[13] M. Clausel and B. Vedel, Explicit construction of operator scaling Gaussian random fields, Fractals, 19 (2011), pp. 101-111.

[14] J. Coeurjolly, Simulation and identification of the fractional brownian motion: A bibliographical and comparative study, J. Stat. Softw, 5 (2000), pp. 1-53.

[15] S. Cohen And J. Istas, Fractional fields and applications, Springer, 2013.

[16] P. Flandrin, Wavelet analysis and synthesis of fractional Brownian motion, IEEE Trans. Inf. Theory, 38 (1992), pp. 910-917.

[17] Z. Gelbaum, Fractional brownian fields over manifolds, T. Am. Math. Soc., 366 (2014), pp. 47814814.

[18] Z. Gelbaum And M. Titus, Simulation of fractional brownian surfaces via spectral synthesis on manifolds, IEEE Trans. Image Process., 23 (2014), pp. 4383-4388.

[19] N. J. Girard And K. Chaumoitre, The brain in the belly: What and how of fetal neuroimaging?, Magn. Reson. Imaging, 36 (2012), pp. 788-804.

[20] D. Hammond, P. Vandergheynst, and R. Gribonval, Wavelets on graphs via spectral graph theory, Appl. Comput. Harmon. Anal., 30 (2011), pp. 129-150.

[21] G. Huisken, Flow by mean curvature of convex surfaces into spheres, Australian National University, Centre for Mathematical Analysis, 1984.

[22] J. Istas, Manifold indexed fractional fields, ESAIM Probab. Stat., 16 (2012), pp. 222-276.

[23] J. ISTAS AND C. LACAUX, On locally self-similar fractional random fields indexed by a manifold, Stochastics An International Journal of Probability and Stochastic Processes, 85 (2013), pp. 489499. 
[24] J. Istas AND G. LANG, Quadratic variations and estimation of the local Hölder index of a Gaussian process, Ann. Inst. Henri Poincaré, Prob. Stat., 33 (1997), pp. 407-436.

[25] A. Kolmogorov, Wienersche spiralen und einige andere interessante kurven im hibertschen raum, Acad.Sci.URSS, 26 (1940), pp. 115-118.

[26] J. Lefèvre, D. Germanaud, J. Dubois, F. Rousseau, I. De Macedo Santos, H. AnGleys, J.-F. Mangin, P. Hüppi, N. Girard, and F. De Guio, Are developmental trajectories of cortical folding comparable between cross-sectional datasets of fetuses and preterm newborns?, Cereb. Cortex, (2015), pp. 1-13.

[27] B. B. Mandelbrot And J. VAn Ness, Fractional Brownian motion, fractional noises and applications, SIAM Rev., 10 (1968), pp. 422-437.

[28] S. E. PAV, Moments of the log non-central chi-square distribution, arXiv:1503.06266 [math, stat], (2015).

[29] R. Peltier And J. Levy Vehel, Multifractional Brownian motion: definition and preliminary results, Tech. Report 2645, INRIA, 1996.

[30] H. Rabiei, F. Richard, O. Coulon, And J. Lefèvre, Local spectral analysis of the cerebral cortex: New gyrification indices, IEEE Trans. Med. Imaging, 36 (2017), pp. 838-848.

[31] H. Rabiei, F. Richard, J. Lefìvre, and O. Coulon, Estimating the complexity of the cerebral cortex folding with a local shape spectral analysis, in Vertex-frequency analysis of graph signals, L. Stanković and E. Sejdić, eds., Springer Nature, 2019.

[32] M. Reuter, S. Biasotti, D. Giorgi, G. Patane, and M. Spagnuolo, Discrete LaplaceBeltrami operators for shape analysis and segmentation, Comput. \& Graph., 33 (2009), pp. 381390.

[33] M. Reuter, F.-E. Wolter, And N. Peinecke, Laplacebeltrami spectra as "shape-DNA" of surfaces and solids, Comput. Aided Des., 38 (2006-04), pp. 342-366.

[34] F. Richard, Analysis of anisotropic Brownian textures and application to lesion detection in mammograms, Procedia Environ. Sci., 27 (2015), pp. 16-20.

[35] F. Richard, Some anisotropy indices for the characterization of Brownian textures and their application to breast images, Spat. Stat., 18 (2016), pp. 147-162.

[36] F. Richard, Tests of isotropy for rough textures of trended images, Stat. Sinica, 26 (2016), pp. 1279-1304.

[37] F. RichaRd, Anisotropy of Hölder Gaussian random fields: characterization, estimation, and application to image textures, Stat. Comput., (2017), pp. 1-14.

[38] F. Richard AND H. Biermé, Statistical tests of anisotropy for fractional Brownian textures. Application to full-field digital mammography, J. Math. Imaging Vis., 36 (2010), pp. 227-240.

[39] D. Shuman, B. Ricaud, And P. Vandergheynst, Vertex-frequency analysis on graphs, Appl. Comput. Harmon. A., 40 (2016), pp. 260-291.

[40] G. Strang And G. J. Fix, An analysis of the finite element method, vol. 212, Prentice-hall Englewood Cliffs, NJ, 1973.

[41] T. Tallinen, J. S. Biggins, and L. Mahadevan, Surface sulci in squeezed soft solids, Phys. Rev. Lett., 110 (2013), p. 024302.

[42] T. Tallinen, J. Y. Chung, F. Rousseau, N. Girard, J. Lefèvre, and L. Mahadevan, On the growth and form of cortical convolutions, Nat. Phys., 12 (2016), pp. 588-593. 
[43] N. Venet, On the existence of fractional brownian fields indexed by manifolds with closed geodesics, arXiv preprint arXiv:1612.05984, (2016).

[44] T. Vu And F. Richard, Statistical tests of heterogeneity for anisotropic multifractional Brownian fields, Stoch. Proc. Appl., (2020), https://doi.org/10.1016/j.spa.2020.01.012. In press.

[45] H. WEYL, Das asymptotische veteilungsgesetz der eigenwerte linearer partieller diffentialgleichungen, Math. ann., 71 (1912), pp. 441-479. 